\title{
Near-Infrared Optical Imaging Noninvasively Detects Acutely Damaged Muscle
}

Stephen M. Chrzanowski, ${ }^{*}$ Abhinandan Batra, ${ }^{\dagger}$ Brittany Lee-McMullen, ${ }^{\ddagger}$ Ravneet S. Vohra, ${ }^{*}$ Sean C. Forbes, ${ }^{\dagger}$ Huabei Jiang, Krista Vandenborne, ${ }^{\dagger}$ and Glenn A. Walter*

From the Departments of Physiology and Functional Genomics, ${ }^{*}$ Physical Therapy, ${ }^{\dagger}$ Biochemistry, ${ }^{\ddagger}$ and Biomedical Engineering, ${ }^{\S}$ University of Florida, Gainesville, Florida

Accepted for publication

June 8, 2016.

Address correspondence to Glenn A. Walter, Ph.D., 1600 S.W. Archer Rd., M552 P.O. Box 100274, Gainesville, FL 32610-0274. E-mail: glennw@ ufl.edu.

\begin{abstract}
Muscle damage is currently assessed through methods such as muscle biopsy, serum biomarkers, functional testing, and imaging procedures, each with its own inherent limitations, and a pressing need for a safe, repeatable, inexpensive, and noninvasive modality to assess the state of muscle health remains. Our aim was to develop and assess near-infrared (NIR) optical imaging as a novel noninvasive method of detecting and quantifying muscle damage. An immobilization-reambulation model was used for inducing muscle damage and recovery in the lower hindlimbs in mice. Confirmation of muscle damage was obtained using in vivo indocyanine green-enhanced NIR optical imaging, magnetic resonance imaging, and ex vivo tissue analysis. The soleus of the immobilized-reambulated hindlimb was found to have a greater amount of muscle damage compared to that in the contralateral nonimmobilized limb, confirmed by in vivo indocyanine green-enhanced NIR optical imaging (3.86-fold increase in radiant efficiency), magnetic resonance imaging (1.41-fold increase in $T_{2}$ ), and an ex vivo spectrophotometric assay of indocyanine green uptake (1.87-fold increase in normalized absorbance). Contrast-enhanced NIR optical imaging provides a sensitive, rapid, and noninvasive screening method that can be used for imaging and quantifying muscle damage and recovery in vivo. (Am J Pathol 2016, 186: 2692-2700; http://dx.doi.org/10.1016/j.ajpath.2016.06.019)
\end{abstract}

\section{Techniques of Assessing Muscle Damage}

Muscle damage is an important and unavoidable outcome of many pathologic states, such as muscular dystrophies, inflammatory myopathies, and physical trauma. Several preclinical models have been developed to induce acute muscle damage, including eccentric loading, ${ }^{1-5}$ immobilizationreloading, ${ }^{6}$ and myotoxin injection. ${ }^{7-10}$ In particular, reloading of muscle following unloading has demonstrated an ability to robustly induce eccentric loading muscle damage. ${ }^{4-6,11}$ Reloading, in the form of reambulation following immobilization, has demonstrated ultrastructural perturbations to muscle consistent with muscle damage. ${ }^{6,11-13}$ Compromised sarcolemmal membranes release muscle enzymes such as creatine kinase while passively taking up large serum proteins and markers such as Evans blue dye (EBD). ${ }^{14}$

Muscle pathology has been measured by a number of techniques, all of which possess their own inherent limitations. These techniques include muscle biopsy, serology, functional measures, and imaging methods. Muscle biopsy, although the most direct measure of pathology, has a capacity too limited to be considered a longitudinal measure of muscle pathology due to the necessity of repeated sample collections. Although serology and functional testing provide a proxy to the overall state of muscle health, they fail to sensitively localize pathology, instead providing information regarding the general health of all muscles in the body, and are complicated by the

Supported by the US Department of Defense project W81XWH-12-10387 (G.A.W.), T32 Neuromuscular Plasticity Training Program project HD043730 (K.V.), and T32 Hypertension Center project HL083810 (PI: Charles Wood). A portion of this work was performed in the McKnight Brain Institute at the National High Magnetic Field Laboratory's AMRIS Facility, which is supported by National Science Foundation Cooperative Agreement No. DMR-1157490 and the State of Florida.

Disclosures: None declared. 
changes in lean body mass typically associated with myopathy. Magnetic resonance imaging (MRI) has evolved as a noninvasive method of detecting and quantifying muscle pathology ${ }^{6,15-19}$ but has several limitations, such as cost; speed of operations; and contraindications in subjects with metallic implants, claustrophobia, and compliance issues. ${ }^{20}$ An attractive possible alternative would be the use of clinically approved fluorescent optical contrast agents for imaging muscle damage in vivo, similar to those currently used for conventional histologic measurements. ${ }^{21-23}$

\section{Near-Infrared Imaging and Indocyanine Green}

In preclinical models of disease, fluorescent optical imaging is a technique widely used for detecting pathology by fluorescent dyes, proteins, and conjugates. ${ }^{24,25}$ By using optical imaging in the near-infrared (NIR) range (700 to $1000 \mathrm{~nm}$ ), two primary advantages exist over conventional fluorophores that operate at shorter wavelengths: deeper photon penetration within tissues and minimal tissue autofluorescence. ${ }^{24,26,27}$ When imaging in the NIR range, penetration of signal can overcome some of the scattering encountered with other fluorescent imaging techniques at shorter wavelengths. ${ }^{28}$

The first, and still the only, NIR fluorescent contrast agent approved by the US Food and Drug Administration (FDA) is indocyanine green (ICG). ${ }^{29}$ ICG is primarily and rapidly bound to albumin and lipoproteins within the circulation and thus acts as a blood-pooling NIR fluorescent agent, highlighting vasculature in several systems. ${ }^{30,31}$ Furthermore, through the enhanced permeation and retention effect, ICG passively accumulates in tumors, in a manner parallel to that of the MR contrast agent gadolinium. ${ }^{32,33}$ Despite its widespread use in imaging other organs, only conjugates of ICG have demonstrated the capability of imaging compromised muscle. ${ }^{22}$ With this in mind, we hypothesized that clinicalgrade ICG will behave similarly to EBD,${ }^{14}$ and accumulate in damaged muscle fibers, allowing for the quantification of muscle damage in a longitudinal and in vivo manner. Importantly, because access to preclinical fluorescent imaging is more ubiquitous than is access to MRI, ICG-enhanced optical imaging may be a suitable complement to more expensive and time-consuming MRI scanners in preclinical muscle pathology studies. In this article we assessed whether ICG-enhanced NIR optical imaging is capable of measuring acute muscle damage and recovery in a targeted deep hindlimb muscle in mice. To validate the NIR optical imaging findings, direct comparisons to MR measures of muscle damage and ex vivo tissue assessments were performed.

\section{Materials and Methods}

\section{Animal Experimental Protocol}

All studies were approved by the University of Florida's institutional animal care and use committee, and the research was performed at the University of Florida, Gainesville, FL. Male
C57BL/6J mice $(n=60)$ were bred in-house through the University of Florida's Animal Care Services and were 6 to 8 weeks of age during experimentation. Mice were housed in a facility regulated by the Association for Assessment and Accreditation of Laboratory Animal Care (12 hours light/dark, $22^{\circ} \mathrm{C}, 42 \%$ humidity) and provided food ad libitum. Additionally, a transgenic dough diet (BioServ, Flemington, NJ) was provided for the mice at the base of the cages during the entire procedure to ensure that dietary needs were met during and after hindlimb immobilization. Right hindlimbs were immobilized in a plantar-flexed position, first by medical-grade paper tape, followed by plaster of Paris (OrthoTape, Blufton, SC), and finally an encompassing single layer of casting material (Patterson Medical, Warrenville, IL), as previously described. ${ }^{6,16}$ The contralateral leg (nonimmobilized) served as each mouse's own control. Mice were checked daily for abrasion wounds as a result of the casting procedure, and animal weight was monitored. After 2 weeks of immobilization, casts were removed, and the animals were allowed to undergo free-cage ambulation. Data (MRI, NIR optical imaging, and tissue assessment) were acquired at $0,1,2,3,5$, and 7 days following the removal of casts ( $n=10$ per time point). Eighteen hours before sacrifice, $1 \%$ filter-sterilized EBD (Sigma-Aldrich, St. Louis, MO) in phosphate-buffered saline $(0.1 \mathrm{~g} / \mathrm{mL}$ per $\mathrm{mg}$ i.p. $)$ was administered to the mice as previously described. ${ }^{14}$

\section{Near-Infrared Optical Imaging}

One hour before NIR optical imaging, NirawaveC ICG (Miltenyi Biotech Inc., San Diego, CA) was administered to the mice according to the package insert ( $1 \mathrm{mg} \mathrm{ICG/kg} \mathrm{body}$ weight i.v.). It was determined that a steady signal was maintained between 30 minutes and 3 hours after injection (data not shown); thus, NIR optical imaging data were collected during this window of time following injections of ICG contrast dye. Mice were anesthetized using an oxygen and isoflurane mixture (3\% induction; $0.75 \%$ to $1 \%$ maintenance) and two-dimensional NIR optical imaging was performed using an In Vivo Fluorescence Imager (PerkinElmer, Waltham, MA). Image capture took, on average, $<1$ minute/ mouse. Acquired images were analyzed using Living Image software version 4.0 (PerkinElmer) on the same In Vivo Fluorescence Imager. Data acquisition was controlled for binning, F/stop, subject height, and field of view. Additionally, normalization to the exposure time of the camera was manually performed for each image. Excitation and emission wavelengths were 745 and $820 \mathrm{~nm}$, respectively. Regions of interest were carefully drawn over both the immobilized and nonimmobilized lower hindlimbs, and total radiant efficiency $\left(\mathrm{p} / \mathrm{cm}^{2} / \mathrm{sr}\right) /\left(\mu \mathrm{W} / \mathrm{cm}^{2}\right)$ within the designated regions of interest was recorded by the In Vivo Fluorescence Imager.

\section{Magnetic Resonance Imaging}

MRI was performed in a $4.7 \mathrm{~T}$ horizontal $22.5-\mathrm{cm}$ bore magnet (Agilent, Santa Clara, CA) immediately following 
NIR optical imaging. Animals were anesthetized using an oxygen and isoflurane mixture (3\% induction; $0.75 \%$ to $1 \%$ maintenance) and were kept warm with a heated water tubing system for the duration of all MR procedures. Respiratory rate and temperature were monitored (Small Animal Instruments, Stony Brook, NY) throughout the scans to ensure adequate physiological maintenance while the animals were under anesthesia, and anesthesia was appropriately adjusted to maintain adequate vital sign values. In all, MRI scans took approximately 30 minutes to perform. The lower hindlimbs of the mice were inserted into a custom-built solenoid ${ }^{1} \mathrm{H}$ coil (200 MHz; 2.0-cm internal diameter). To obtain correct positioning of all subsequent scans, localizer images in three orthogonal planes were acquired using a gradient echo sequence (repetition time $=30 \mathrm{~ms}$; echo time $=5 \mathrm{~ms}$; slice thickness $=2 \mathrm{~mm}$; slice number $=3$ per plane; acquisition matrix $=128 \times 128$; signal averages $=1$ ). Axial proton $\mathrm{T}_{2^{-}}$ weighted multislice MRIs were acquired along the length of all lower hindlimbs (repetition time $=2000 \mathrm{~ms}$; echo time $=$ 14 and $40 \mathrm{~ms}$; field of view $=10 \times 20 \mathrm{~mm}^{2}$; slice thickness $=1 \mathrm{~mm}$; slice number $=12$; acquisition matrix $=128 \times 256$; signal averages $=2$ ). Signal-to-noise ratios were approximately $21: 1$ and 7.4:1 at echo times of 14 and $40 \mathrm{~ms}$, respectively. MRIs were converted from raw Varian format to digital imaging and communications in medicine files for analysis. Series of three consecutive slices were used for quantifying MRI-T ${ }_{2}$ measurements, beginning approximately $6 \mathrm{~mm}$ distal to the tibial plateau as the anatomic reference point, ensuring that consistent measurements were performed. Regions of interest around the soleus, gastrocnemius, and tibialis anterior muscles were drawn using OsiriX software version 5.0.2 (OsiriX, Geneva, Switzerland) to calculate signal intensity, and the mean $T_{2}$ relaxation times of each of the designated muscles was calculated from the pixel signal intensity at echo times of 14 and $40 \mathrm{~ms}$, as previously described. ${ }^{6,16,18,34}$

\section{Histologic Examination and Spectrophotometric Quantification of ICG and EBD}

Mice were sacrificed the day following NIR optical imaging and MR data capture. At 15 to 18 hours before animal sacrifice, mice were administered EBD and ICG. Muscles (tibialis anterior, soleus, and gastrocnemius) were carefully dissected, fixed in optimal cutting temperature medium at resting length, and immediately frozen in precooled isopentane, then liquid nitrogen, and stored at $-80^{\circ} \mathrm{C}$. Morphologic features of captured tissue sections were assessed by hematoxylin and eosin staining, and uptake of EBD into fibers was determined by fluorescent microscopy. To determine the percentage of muscle that was EBD positive, fibers that stained positive for EBD were manually counted and divided by the total number of fibers in each muscle's cross-sectional area.

Whereas muscle fiber accumulation of EBD could be easily visualized using standard epifluorescence of cryosectioned muscle, attempts to directly visualize ICG in tissue sections were unsuccessful despite the use of a highsensitivity back-thinned charge coupled device (catalog number EM-CCD C9100-13; Hamamatsu, Hamamatsu City, Japan) due to ICG concentration limitations. Therefore, spectrophotometric quantification of ICG and EBD accumulation in individual muscles (soleus, gastrocnemius, and tibialis anterior) was performed as previously described. ${ }^{35}$ In brief, muscles were pulverized in lyzing matrix D tubes (MP Biomedicals, Santa Ana, CA) in dimethyl sulfoxide (Sigma-Aldrich, St. Louis, MO), followed by centrifugation. The absorbance values of EBD and ICG were subsequently measured at 620 and $780 \mathrm{~nm}$, respectively, on a SpectraMax 5 spectrophotometer (Molecular Devices, Sunnyvale, CA), and normalized to tissue weights for assessment of dye uptake.

\section{Statistical Analysis}

All statistical analyses were performed using Prism software version 6.0 (GraphPad, La Jolla, CA). Results are expressed as means \pm SEM, unless otherwise stated. Significance levels were tested at an $\alpha$ level of 0.05 . Noncasted hindlimbs served as the control for each individual animal. Two-way analysis of variance with planned Bonferroni correction was performed to compare both the duration of reambulation and the presence or absence of immobilization. Cohen's d values were calculated to determine effect sizes for comparing NIR optical imaging with MRI. A linear regression model was used for calculating the correlation between radiant efficiency, MRI-T $\mathrm{T}_{2}$, and spectrophotometric data.

\section{Results}

\section{Animal Procedures}

Throughout the duration of the experiments, all mice were maintained at a body weight within $10 \%$ of preimmobilization weight, and three needed recasting because abrasive lesions had developed on the skin. In these rare cases, topical antibiotics were applied to address abrasive lesions, all with resolution. One mouse unexpectedly expired following data collection in the MR scanner and was not used in the data analysis. The single hindlimb casting procedures were otherwise well-tolerated through the duration of the experiments.

\section{Near-Infrared Imaging of Mouse Hindlimbs}

Before the immobilization procedure, mice demonstrated minimal fluorescence distribution in both hindlimbs, and on comparison of preimmobilization and day- 0 reambulated hindlimbs, no differences were observed between the immobilized and nonimmobilized hindlimbs (Figure 1A). Throughout the reambulation phase, radiant efficiency in the immobilized-reambulated hindlimbs significantly peaked by day 2 and was 3.86-fold higher than precasted values, 

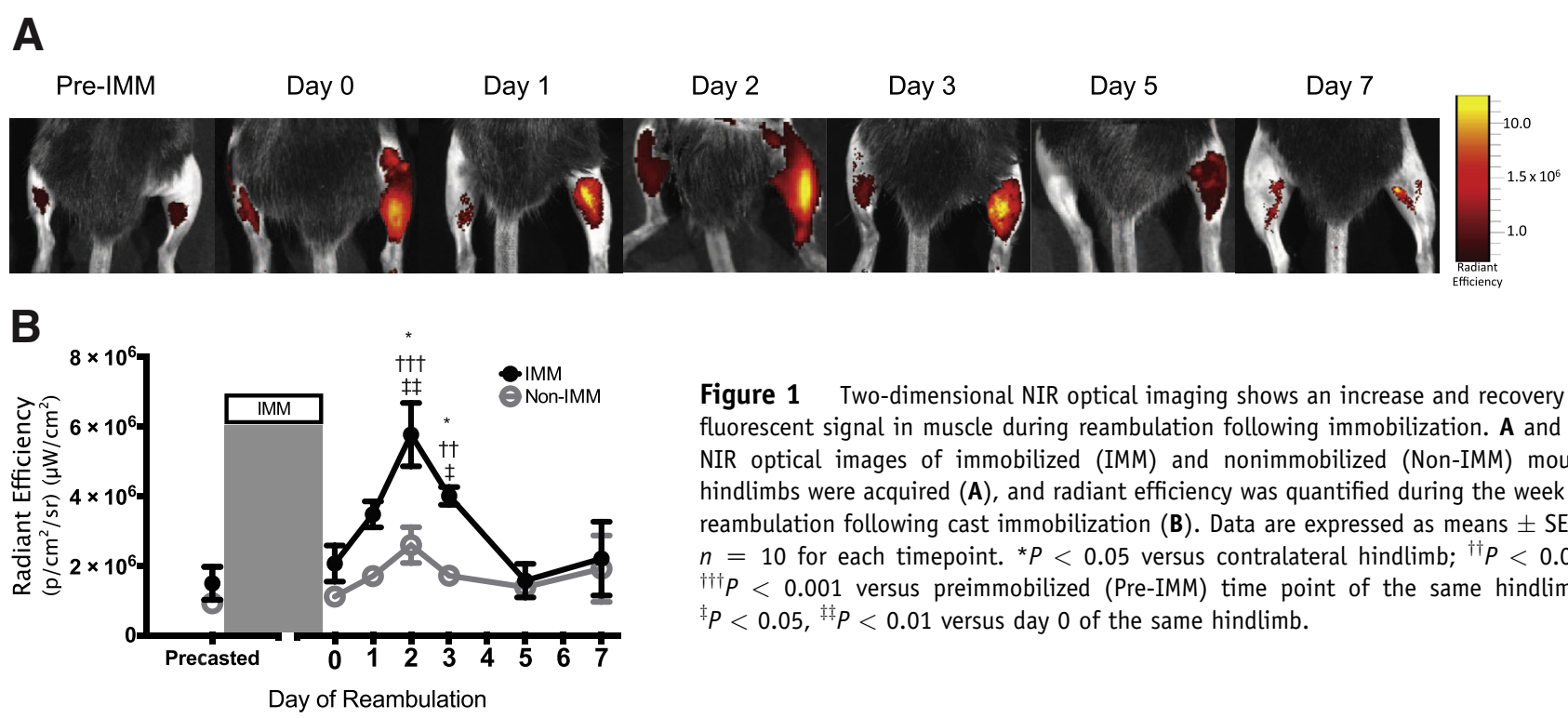

\begin{abstract}
Figure 1 Two-dimensional NIR optical imaging shows an increase and recovery of fluorescent signal in muscle during reambulation following immobilization. A and $\mathbf{B}$ : NIR optical images of immobilized (IMM) and nonimmobilized (Non-IMM) mouse hindlimbs were acquired $(\mathbf{A})$, and radiant efficiency was quantified during the week of reambulation following cast immobilization (B). Data are expressed as means $\pm \mathrm{SEM}$. $n=10$ for each timepoint. ${ }^{*} P<0.05$ versus contralateral hindlimb; ${ }^{\dagger} P<0.01$, ${ }^{\dagger \dagger} P<0.001$ versus preimmobilized (Pre-IMM) time point of the same hindlimb; ${ }^{\ddagger} P<0.05,{ }^{\ddagger \ddagger} P<0.01$ versus day 0 of the same hindlimb.
\end{abstract}

followed by a return to baseline by day 7 (Figure 1). Interestingly, the contralateral hindlimbs also demonstrated an increase (2.45-fold) in total radiant efficiency between day 2 reambulation and precasted values, but this difference did not reach significance. NIR images are presented (Figure 1A), allowing for qualitative demonstration of the immobilized (right) versus nonimmobilized (left) hindlimbs. The quantitative assessment time course of total radiant efficiency throughout the week of reambulation is presented in Figure 1B. The total radiant efficiency values of the immobilized-reambulated hindlimbs on days 2 and 3 of reambulation were significantly different from those of the contralateral and preimmobilized hindlimbs and day 0 of the immobilized-reambulated limbs. Interestingly, the nonimmobilized hindlimbs demonstrated a subtle, nonsignificant increase in total radiant efficiency on the 2nd day of reambulation.

\section{Magnetic Resonance Imaging Confirms Muscle Damage after Cast Immobilization and Reambulation}

Preimmobilization, both hindlimbs demonstrated homogenous contrast in all of the muscles on $\mathrm{T}_{2}$-weighted MRIs (Figure 2A). The soleus muscle of the immobilizedreambulated hindlimb demonstrated the greatest $T_{2}$ changes, peaking at 2 days following cast removal (1.41-fold change increase in $\mathrm{T}_{2}$ ), and values were comparable to those from baseline by day 5 of the reambulation phase (Figure 2B). Interestingly, both the gastrocnemii and tibialis anterior muscles of the immobilized-reambulated hindlimbs demonstrated subtle, yet significant, differences in $T_{2}$ changes (1.13- and 1.14-fold changes, respectively) compared with those of their contralateral nonimmobilized limbs at the initiation of reambulation, but these values were not significantly different from preimmobilization measures (Figure 2, C and D).

\section{Histologic Findings}

Ex vivo assessment of tissue was used for confirming in vivo NIR optical imaging and MRI findings. The appearance of EBD accumulation at both the microscopic and macroscopic levels within the soleus muscles confirmed the well-established time course of damage and recovery during reloading following immobilization. EBD was minimally taken up into soleus muscle fibers immediately following cast removal (Figure 3A). Quantitative demonstration of dye uptake into the immobilized-reambulated and contralateral control solei are demonstrated in Figure 3B. The percentage of EBD-positive fibers in the gastrocnemius (Supplemental Figure S1) was elevated at day 3 relative to baseline values; the tibialis anterior (Supplemental Figure S2) demonstrated no significant changes in EBD-positive fibers throughout the reambulation period. By the 2nd day of reambulation, fibers of the immobilized-reambulated soleus appeared, with $47.1 \% \pm 15.6 \%$ of the fibers being EBD positive in a checkerboard pattern. EBD uptake into the contralateral soleus was less, with $15.1 \% \pm 6.3 \%$ of fibers the being EBD positive, but this value was not significantly different from that on day 0 in the soleus muscle in the same control limb. At the end of the reambulation week, EBD signal is again less visible with only $5.6 \% \pm 2 \%$ and $1.6 \% \pm 1.8 \%$ of the fibers being EBD positive for the immobilized-reambulated and control hindlimbs, respectively.

Hematoxylin and eosin-stained sections of the immobilized and reambulated soleus at various time points throughout the week demonstrated the well-characterized histopathologic features of muscle damage, most noticeably on the 2nd day of reambulation. ${ }^{6}$ At the peak of damage (day 2), widened extracellular spaces, decreased density of muscle fibers, and variability in fiber size were all qualitatively observed in the soleus. In the soleus, the percentage of myonecrotic pale fibers was nonsignificantly 


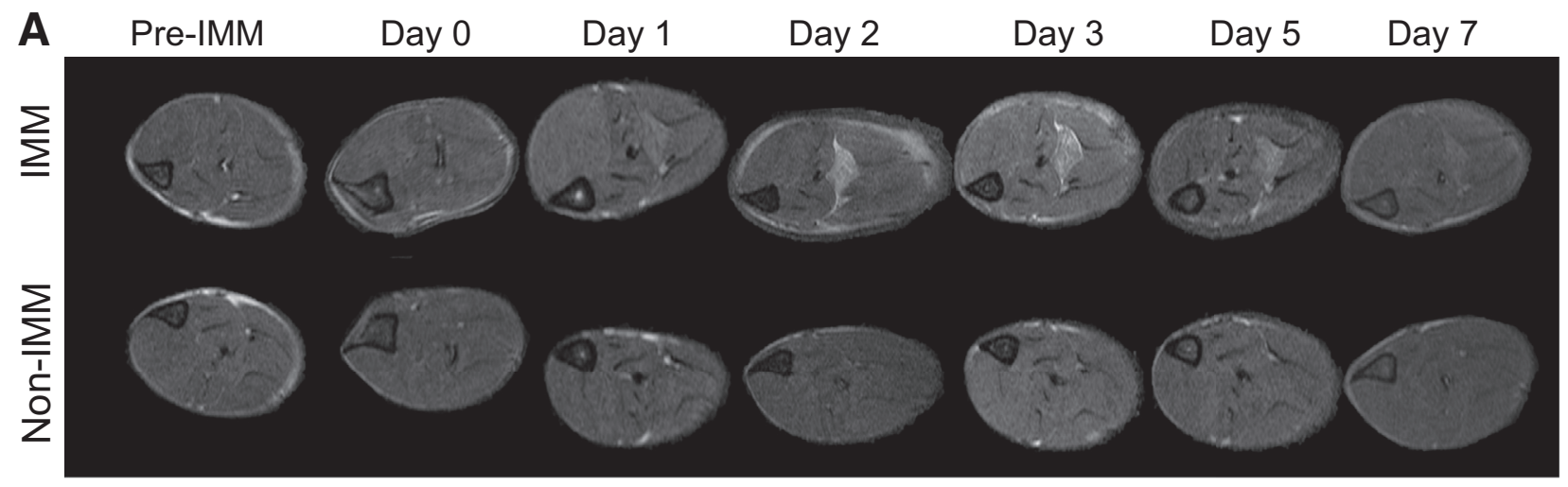

B

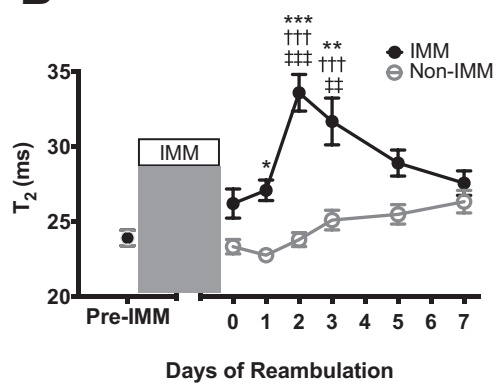

C

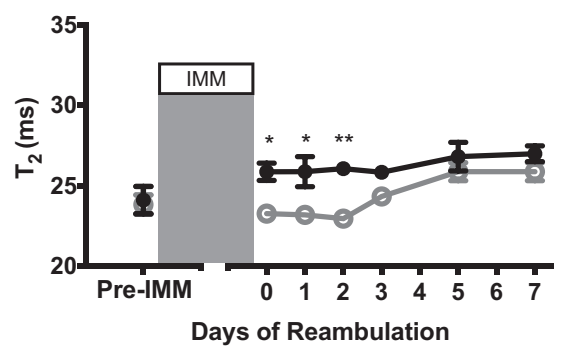

D

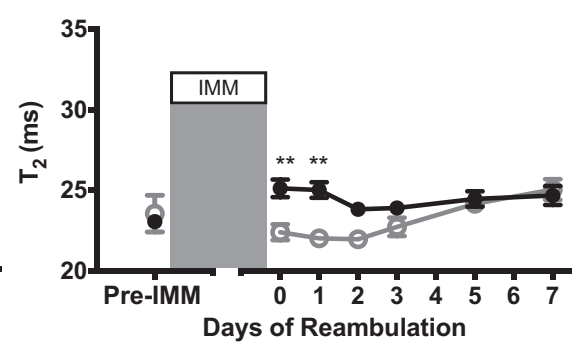

Figure 2 MRI- $T_{2}$ shows damage and recovery of soleus, but not gastrocnemius or tibialis anterior, muscles during reambulation. A: Representative crosssectional $T_{2}$ spin echo MR images are shown throughout reambulation following single hindlimb casting. B-D: The immobilization (IMM) procedure causes initial differences in the soleus (B), gastrocnemius (C), and tibialis anterior (D), but only the soleus' $T_{2}$ values continue to increase by the second day of reambulation, followed by recovery to preimmobilization (Pre-IMM) levels. Data are expressed as means \pm SEM. $n=10$ for each timepoint. ${ }^{*} P<0.05$, ${ }^{* *} P<0.01$, and ${ }^{* * *} P<0.001$ versus contralateral hindlimb; ${ }^{\dagger \dagger \dagger} P<0.001$ versus preimmobilization time point of the same hindlimb; ${ }^{\ddagger \ddagger} P<0.01,{ }^{\ddagger \ddagger \ddagger} P<0.001$ versus day 0 of the same hindlimb. Non-IMM, nonimmobilized.

increased in the immobilized hindlimbs on days 1 and 2 (Supplemental Figure S3A), the percentage of fibers undergoing myophagocytosis was significantly increased on day 1 (Supplemental Figure S3B), and the percentage of basophilic regenerating fibers was significantly elevated on days 5 and 7 (Supplemental Figure S3C). Analysis of H\&E sections of the gastrocnemii (Supplemental Figure S1). The tibialis anterior (Supplemental Figure S2) demonstrated a

A Days of Reambulation

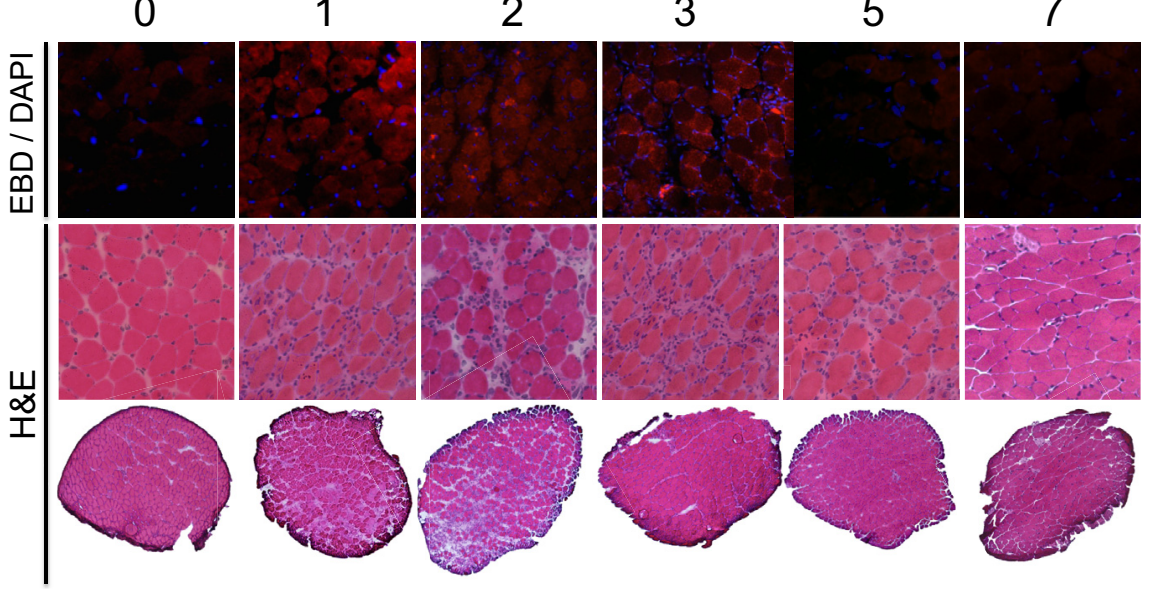

B

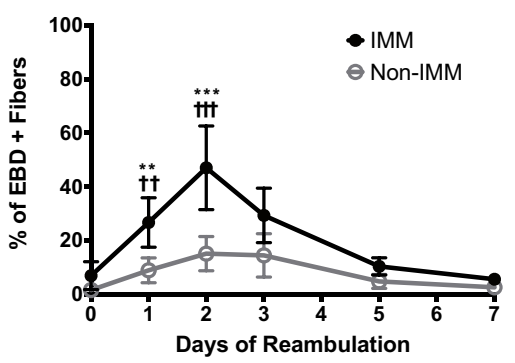

Figure 3 Histologic assessment confirms damage and recovery in the reambulated soleus muscle of the immobilized-reambulated hindlimb at the second day of reambulation. A: Fluorescent uptake of Evans blue dye (EBD; red) with surrounding myonuclei stained by DAPI (blue; top row). Representative sections (middle row) and whole soleus sections (bottom row) stained with hematoxylin and eosin (H\&E) are also shown. B: The percentages of fibers that stained positive for EBD throughout the week of reambulation in the immobilized (IMM) and nonimmobilized (Non-IMM) solei muscles. Data are expressed as means \pm SEM. $n=10$ for each timepoint. ${ }^{* *} P<0.01,{ }^{* *} P<0.001$ versus contralateral hindlimb; ${ }^{\dagger \dagger} P<0.01,{ }^{\dagger \dagger \dagger} P<0.001$ versus day 0 of the same hindlimb. Original magnification: $\times 40$ (top row and middle row, A); $\times 10$ (bottom row, A). 
quantitative lack of acute myonecrotic pale fibers, percentage of fibers undergoing myophagocytosis, and basophilic regenerating fibers (Supplemental Figures S4 and S5).

\section{Spectrophotometric Quantification of Accumulation of ICG and EBD within Muscle}

Muscle lysates were analyzed by a spectrophotometer to quantify both EBD and ICG accumulation within each lower leg muscle throughout the week of reambulation (Figure 4). Absorbance, normalized to muscle weight, was determined at $620 \mathrm{~nm}$ (EBD) (Figure 4A) and $780 \mathrm{~nm}$ (ICG) (Figure 4B) and demonstrated significant peaks in signal at the 2nd day of reambulation with both dyes (EBD, 1.72-fold change; ICG, 1.87-fold change), followed by a return to baseline values by the end of the week of reambulation (EBD, 1.21-fold change; ICG, 1.20-fold change). Absorbance of EBD and ICG in the gastrocnemius (Supplemental Figure S6) and tibialis anterior (Supplemental Figure S7) lysates were not significantly elevated as compared to background noise levels, indicating minimal dye uptake per milligram of tissue weight into these two muscle groups.

\section{Correlation between MR and Optical Imaging}

To determine whether a correlation exists between muscle damage measures and total radiant efficiency, $M R I-\mathrm{T}_{2}$ and spectrophotometric absorbance values were compared to total radiant efficiency at the peak of muscle damage. Figure 5 shows the linear relationships between radiant efficiency and MRI-T $T_{2}$ in the immobilized-reambulated and control muscles. Table 1 quantitatively shows the significance of linear regression correlations between NIR optical imaging radiant efficiency compared to MRI- $\mathrm{T}_{2}$ and optical density $780 \mathrm{~nm} / \mathrm{mg}$ tissue. Significant correlations (Table 1) were demonstrated only when the solei measures were compared, and neither the gastrocnemii nor the tibialis anterior muscles demonstrated any significant correlations with the total radiant efficiency. Finally, to determine the robustness of each imaging modality, Cohen's d effect sizes were calculated to determine the magnitude of the difference between days 0 and 2 in the immobilized-reambulated hindlimbs. The effect sizes of MRI-T $\mathrm{T}_{2}$ and NIR optical imaging were 1.79 and 1.57 , respectively, suggesting comparable magnitudes of differences between the two imaging modalities.

\section{Discussion}

The main purpose of this study was to demonstrate proof-ofprinciple findings that an FDA-approved NIR fluorescent contrast agent (ICG) and NIR optical imaging can noninvasively image muscle in a well-characterized model of acute muscle damage and recovery. Muscle damage and

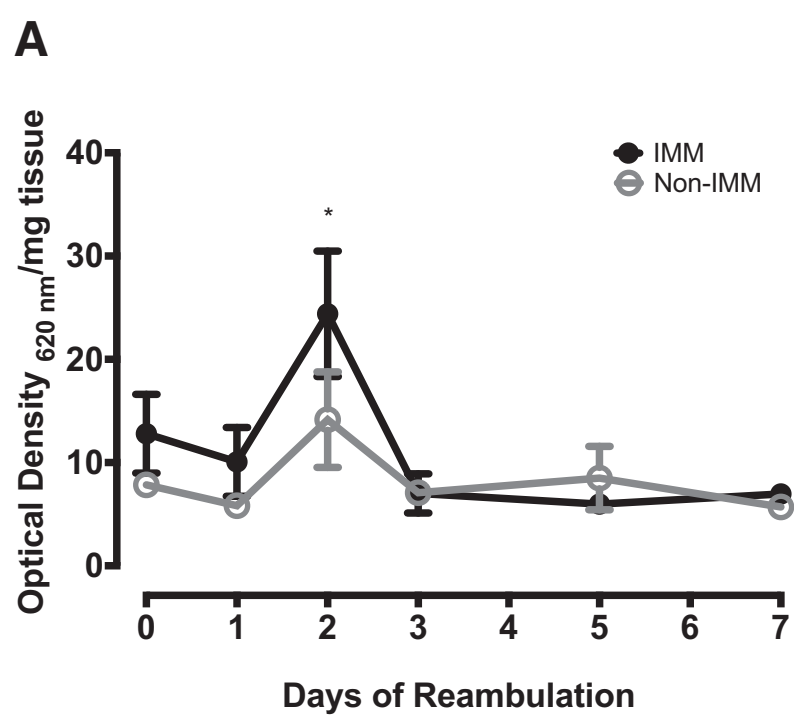

B

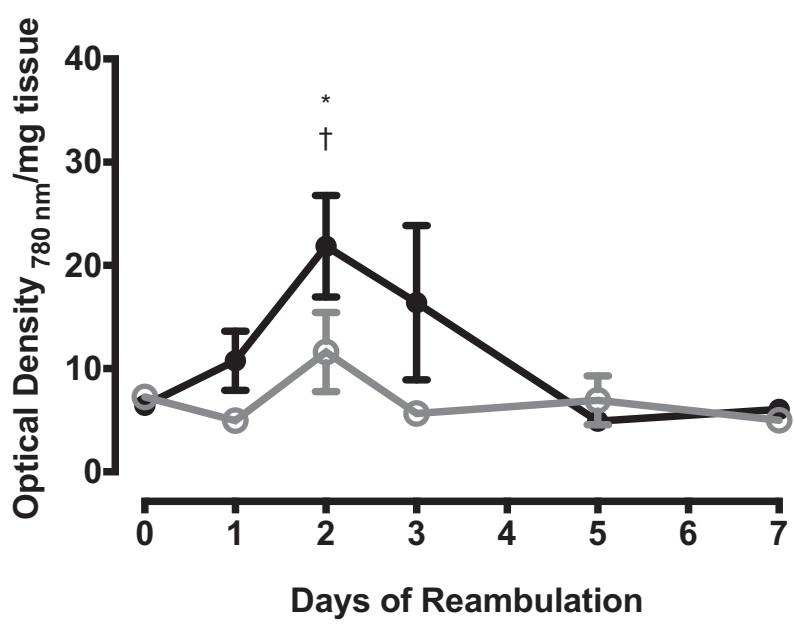

Figure 4 Spectrophotometric assessment confirms dye uptake into the soleus muscle at the peak of muscle damage. Absorbance values measured at 620 and $780 \mathrm{~nm}$, measuring Evans blue dye (A) and indocyanine green (B), respectively, demonstrate that both dyes are taken up into the soleus muscle of the immobilized (IMM)/reambulated hindlimb, significantly peaking at the second day of reambulation, followed by a return back to baseline values. Data are expressed as means \pm SEM. $n=10$ for each timepoint. ${ }^{*} P<0.05$ versus contralateral hindlimbs; ${ }^{\dagger} P<0.05$ versus day 0 of the same hindlimb. Non-IMM, nonimmobilized.

recovery in the soleus muscle of immobilized-reambulated mouse hindlimbs were visualized and quantified using ICGenhanced NIR optical imaging, with further supporting confirmation provided by MRI- $\mathrm{T}_{2}$, histologic examination, and spectrophotometric assessments. The time course of muscle damage and recovery following immobilization and free reambulation using both imaging modalities agreed with findings from histologic and biochemical analyses of the extracted tissues. This study demonstrates the ability of ICG-enhanced NIR optical imaging to two-dimensionally visualize and quantify muscle damage in vivo in a safe, quantitative, and longitudinal manner. 


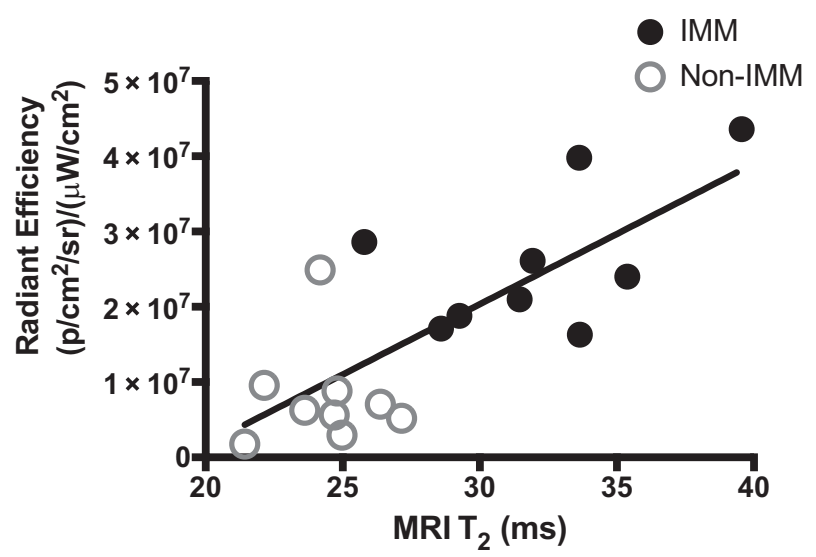

Figure 5 Increased radiant efficiency correlates to increased markers of damage in the soleus muscle. At the second day of reambulation following immobilization (IMM), individual mice with increased radiant efficiency values also concurrently have increased MRI $T_{2}$ visually separating the nonimmobilized (Non-IMM) hindlimbs from the immobilized hindlimbs.

ICG was chosen as the NIR fluorescent blood-pooling agent because its properties are similar to those of $\mathrm{EBD}^{14}$ in that it binds to serum albumin and was hypothesized to accumulate in damaged muscle cells. We exploited the fact that optical imaging in the NIR range allows for deep tissue imaging and minimal tissue autofluorescence, allowing for imaging of the deepest muscle of the mouse hindlimb. ${ }^{24}$ Additionally, NIR optical imaging has the advantage of complementing current MR techniques, ${ }^{33}$ with the additional advantage that data acquisition can be achieved in a more cost- and time-efficient manner. Although NIR optical imaging of ICG has been demonstrated clinically in other tissues of the body, the primary use of NIR optics has been to perform NIR spectroscopic analyses of changes in perfusion status in muscle, although Inage et $\mathrm{al}^{22}$ demonstrated the ability to image muscle damage in a rat model using an ICG conjugate.

We sought to quantify and visualize muscle damage through mouse hindlimb immobilization followed by reambulation, which is known to cause damage to muscle. ${ }^{6}$ For this study, this model of hindlimb immobilization was chosen for testing the ability of NIR optical imaging to

Table 1 The Solei Correlates with NIR Optical Imaging Signal More Strongly than Does Either the Gastrocnemii or Tibialis Anterior

\begin{tabular}{llc}
\hline Muscle & MRI-T $_{2}$ & Absorbance $_{780 \mathrm{~nm} / \mathrm{mg} \text { tissue }}$ \\
\hline Sol & $0.57^{* * *}$ & $0.46^{* *}$ \\
Gas & 0.21 & 0.001 \\
TA & 0.12 & $<0.001$ \\
\hline
\end{tabular}

NIR optical imaging radiant efficiency measures were correlated with MRI- $T_{2}$ and an optical density of $780 \mathrm{~nm} / \mathrm{mg}$ tissue. $R^{2}$ and $P$ values are shown.

${ }^{* *} P<0.01,{ }^{* * *} P<0.001$

Gas, gastrocnemii; NIR, near-infrared; Sol, solei; TA, tibialis anterior. detect and quantify muscle damage as the technique demonstrates specific damage to the soleus. This model of muscle damage is well-characterized to disrupt the sarcolemma of the slow-twitch soleus muscle specifically, demonstrated by uptake of EBD. ${ }^{6,36}$ Furthermore, the time course of muscle damage and recovery is well-characterized in this model, and contralateral noncasted hindlimbs allow for internal controls within each mouse. ${ }^{6}$ NIR optical images were used for visualizing muscle damage, which was confirmed on MRI-T $\mathrm{T}_{2}$, histologic examination, and ICG/ EBD accumulation in isolated muscles. Importantly, a return to baseline levels of all measures of muscle damage was observed, indicating that NIR optical imaging can be used for imaging both damage and recovery of muscle. By comparing the effect sizes of MRI-T $\mathrm{T}_{2}(\mathrm{~d}=1.79)$ and NIR optical imaging $(\mathrm{d}=1.57)$, we determined that NIR imaging had an ability to detect muscle damage similar to that of MRI. Additionally, we found a significant linear relationship between NIR optical imaging and MRI-T (Figure 5), further confirming NIR optical imaging as a capable noninvasive modality of detecting muscle damage.

Because of significant correlations between NIR optical imaging measures to MRI-T $\mathrm{T}_{2}$ and preferential tissue accumulation of EBD/ICG in the soleus, it can be hypothesized that the NIR optical imaging signal is predominantly due to the soleus, rather than either the gastrocnemius or tibialis anterior. Lastly, biochemical analysis of tissue EBD and ICG accumulation at the peak of muscle damage confirmed that, in the soleus, ICG content values per milligram of tissue weight were 12.8- and 5.2-fold greater than in the gastrocnemius and tibialis anterior, respectively. The disproportionate uptake of ICG into the soleus indicates that the immobilized-reambulated soleus (Figure 4B) most likely is the primary contributor to fluorescent signal, as seen in Figure 1.

It is important to consider that MR- and ICG-enhanced NIR optical imaging procedures assess different properties of tissue, with MR assessing inherent magnetic properties of tissue and NIR optical imaging assessing vascular perfusion and membrane stability. Although MRI-T ${ }_{2}$ hyperintensities clearly identified the damaged soleus muscle, elevated $T_{2}$ signals may be caused by a number of mechanisms, including lipid infiltration or edematous inflammation within muscle. Importantly, our study demonstrated that muscle damage results in not only elevated MRI- $T_{2}$ values but also elevated radiant efficiency, as observed by ICGenhanced NIR optical imaging. Because C57BL/6J mice do not have lipid deposited within muscle, the elevated MRI-T ${ }_{2}$ observed in the soleus was not due to lipid deposition and was likely due to inflammatory edema, which is well-established in reloaded muscle. ${ }^{11-13}$ Interestingly, we saw increases of MRI-T $\mathrm{T}_{2}$ signal from days 0 to 2 of reambulation in the gastrocnemius and tibialis anterior muscles. Although histologic findings did not suggest evidence of premyonecrotic pathology (Supplemental Figures S4 and S5), the immobilization procedure may have led to 
elevated MRI- $\mathrm{T}_{2}$ values in the gastrocnemius (Figure 2C) and tibialis anterior (Figure 2D). This phenomenon has also been observed in MRI studies in humans, during lower leg immobilization following ankle fracture. ${ }^{37}$ As described by Caron et al, ${ }^{38}$ contralateral nonimmobilized limbs experience an overload of stimuli, which may explain the nonsignificant, but observable, increase in total radiant efficiency observed on day 2 in the nonimmobilized hindlimb (Figure 1). For these reasons, it is suggested that NIR optical imaging should be used for complementing, rather than supplementing, MR technology, as it may provide additional information in a cost- and time-efficient manner.

ICG, as a nontargeted contrast agent, demonstrated both advantages and disadvantages in this study. An advantage was that it could be used for quantitatively assessing muscle damage and recovery. Because it is not specific to the pathology induced by the immobilization-reambulation technique used in this study, it can theoretically be applied to other pathologies and diseases affecting muscle. Although ICG-albumin uptake is nonspecific, with future modifications, it may provide a platform for targeting specific cell moieties to add further diagnostic and therapeutic value. ${ }^{39,40}$ Importantly, when performing NIR optical imaging without ICG, no elevation of radiant efficiency was observed and signal was comparable to that of background noise (Supplemental Figure S8). Another limitation of contrast-enhanced NIR optical imaging is the lack of spatial sensitivity. Although NIR optical imaging was deemed comparably sensitive to MR techniques of detecting muscle damage by magnitude of effect size assessment, additional technology development should be pursued to increase spatial sensitivity of NIR optical imaging. Future experiments are planned to optimize spatial sensitivity of NIR optical imaging, as well as to test other models of muscle damage, such as myotoxin treatments and preclinical models of muscular dystrophy. As this study demonstrates, ICG-enhanced NIR optical imaging is able to image acutely damaged muscle, which may allow it to be an appropriate diagnostic tool for assessing muscle damage resulting from the spectrum of myositis disorders, muscular dystrophies, exercise studies, and other pathologies that lead to damaged muscle.

Overall, we describe the feasibility of using a technology (NIR optical imaging) with an FDA-approved fluorescent contrast dye (ICG) for quantitatively imaging acute muscle damage and recovery in a well-characterized mouse model. Because of the cost-effectiveness, lack of ionizing radiation or radioactive substrates, and longitudinal capabilities, NIR optical imaging can be used for a diverse range of purposes. ${ }^{21,22,41}$ By using a clinically approved contrast dye with NIR optical imaging, a multipurpose, noninvasive, and safe imaging technology, it is anticipated that this technology can be expeditiously applied to other preclinical models of muscle diseases to complement, rather than supplement, conventional imaging modalities.

\section{Acknowledgments}

We thank William Triplett and Matthew Lapa for assistance with imaging analysis, Huadong Zeng for assistance with operating the MRI scanner, and Drs. Yanhua Deng and Hao Yang for assistance in the NIR optical imaging portions of this study.

\section{Supplemental Data}

Supplemental material for this article can be found at http://dx.doi.org/10.1016/j.ajpath.2016.06.019.

\section{References}

1. Armstrong RB, Ogilvie RW, Schwane JA: Eccentric exercise-induced injury to rat skeletal muscle. J Appl Physiol Respir Environ Exerc Physiol 1983, 54:80-93

2. Clarkson PM, Hubal MJ: Exercise-induced muscle damage in humans. Am J Phys Med Rehabil 2002, 81:S52-S69

3. Clarkson PM, Byrnes WC, McCormick KM, Turcotte LP, White JS: Muscle soreness and serum creatine kinase activity following isometric, eccentric, and concentric exercise. Int J Sports Med 1986, 7: $152-155$

4. Ploutz-Snyder LL, Tesch PA, Hather BM, Dudley GA: Vulnerability to dysfunction and muscle injury after unloading. Arch Phys Med Rehabil 1996, 77:773-777

5. Proske U, Morgan DL: Muscle damage from eccentric exercise: mechanism, mechanical signs, adaptation and clinical applications. J Physiol 2001, 537:333-345

6. Frimel TN, Walter GA, Gibbs JD, Gaidosh GS, Vandenborne K: Noninvasive monitoring of muscle damage during reloading following limb disuse. Muscle Nerve 2005, 32:605-612

7. Gutiérrez JM, Ownby CL: Skeletal muscle degeneration induced by venom phospholipases A2: insights into the mechanisms of local and systemic myotoxicity. Toxicon 2003, 42:915-931

8. Lomonte B, Gutiérrez JM: A new muscle damaging toxin, myotoxin II, from the venom of the snake Bothrops asper (terciopelo). Toxicon 1989, 27:725-733

9. Lomonte B, Tarkowski A, Hanson LA: Host response to Bothrops asper snake venom. Analysis of edema formation, inflammatory cells, and cytokine release in a mouse model. Inflammation 1993, 17:93-105

10. Lomonte B, Angulo Y, Calderón L: An overview of lysine-49 phospholipase A2 myotoxins from Crotalid snake venoms and their structural determinants of myotoxic action. Toxicon 2003, 42:885-901

11. Kasper CE: Sarcolemmal disruption in reloaded atrophic skeletal muscle. J Appl Physiol (1985) 1995, 79:607-614

12. Krippendorf BB, Riley DA: Temporal changes in sarcomere lesions of rat adductor longus muscles during hindlimb reloading. Anat Rec 1994, 238:304-310

13. Vijayan K, Thompson JL, Riley DA: Sarcomere lesion damage occurs mainly in slow fibers of reloaded rat adductor longus muscles. J Appl Physiol (1985) 1998, 85:1017-1023

14. Hamer PW, McGeachie JM, Davies MJ, Grounds MD: Evans Blue Dye as an in vivo marker of myofibre damage: optimising parameters for detecting initial myofibre membrane permeability. J Anat 2002, 200:69-79

15. Dunn JF, Zaim-Wadghiri Y: Quantitative magnetic resonance imaging of the mdx mouse model of Duchenne muscular dystrophy. Muscle Nerve 1999, 22:1367-1371

16. Frimel TN, Kapadia F, Gaidosh GS, Li Y, Walter GA, Vandenborne K: A model of muscle atrophy using cast immobilization in mice. Muscle Nerve 2005, 32:672-674 
17. Kobayashi YM, Rader EP, Crawford RW, Iyengar NK, Thedens DR, Faulkner JA, Parikh SV, Weiss RM, Chamberlain JS, Moore SA, Campbell KA: Sarcolemma-localized nNOS is required to maintain activity after mild exercise. Nature 2008, 456:511-515

18. Vohra RS, Mathur S, Bryant ND, Forbes S, Vandenborne K, Walter GA: Age-related T2 changes in hindlimb muscles of mdx mice. Muscle Nerve 2016, 53:84-90

19. Walter G, Cordier L, Bloy D, Lee Sweeney H: Noninvasive monitoring of gene correction in dystrophic muscle. Magn Reson Med 2005, 54:1369-1376

20. Brockmann MA, Kemmling A, Groden C: Current issues and perspectives in small rodent magnetic resonance imaging using clinical MRI scanners. Methods 2007, 43:79-87

21. Baudy AR, Sali A, Jordan S, Kesari A, Johnston HK, Hoffman EP, Nagaraju K: Non-invasive Optical Imaging of Muscle Pathology in mdx Mice Using Cathepsin Caged Near-Infrared Imaging. Mol Imaging Biol 2011, 13:462-470

22. Inage K, Sakuma Y, Yamauchi K, Suganami A, Orita S, Kubota G, Oikawa Y, Sainoh T, Sato J, Fujimoto K, Shiga Y, Takahashi K, Ohtori S, Tamura T: Longitudinal evaluation of local muscle conditions in a rat model of gastrocnemius muscle injury using an in vivo imaging system. J Orthop Res 2015, 33:1034-1038

23. Kossodo S, Pickarski M, Lin SA, Gleason A, Gaspar R, Buono C, Ho G, Blusztajn A, Cuneo G, Zhang J, Jensen J, Hargreaves R, Coleman P, Hartman G, Rajopadhye M, Duong LY, Sur C, Yared W, Peterson J, Bohumil B: Dual in vivo quantification of integrin-targeted and protease-activated agents in cancer using fluorescence molecular tomography (FMT). Mol Imaging Biol 2010, 12:488-499

24. Frangioni JV: In vivo near-infrared fluorescence imaging. Curr Opin Chem Biol 2003, 7:626-634

25. Tan Y, Jiang H: Diffuse optical tomography guided quantitative fluorescence molecular tomography. Appl Opt 2008, 47:2011-2016

26. Weissleder R: A clearer vision for in vivo imaging. Nat Biotechnol 2001, 19:316-317

27. Weissleder R, Ntziachristos V: Shedding light onto live molecular targets. Nat Med 2003, 9:123-128

28. Ntziachristos V, Ripoll J, Wang LV, Weissleder R: Looking and listening to light: the evolution of whole-body photonic imaging. Nat Biotechnol 2005, 23:313-320

29. Alford R, Simpson HM, Duberman J, Hill GC, Ogawa M, Regino C, Kobayashi H, Choyke PL: Toxicity of organic fluorophores used in molecular imaging: literature review. Mol Imaging 2009, 8:341-354
30. Kobayashi S, Ishikawa T, Tanabe J, Moroi J, Suzuki A: Quantitative cerebral perfusion assessment using microscope-integrated analysis of intraoperative indocyanine green fluorescence angiography versus positron emission tomography in superficial temporal artery to middle cerebral artery anastomosis. Surg Neurol Int 2014, 5:135

31. Raabe A, Beck J, Gerlach R, Zimmermann M, Seifert V: Near-infrared indocyanine green video angiography: a new method for intraoperative assessment of vascular flow. Neurosurgery 2003, 52:132-139

32. Corlu A, Choe R, Durduran T, Rosen MA, Schweiger M, Arridge SR, Schnall MD, Yodh AG: Three-dimensional in vivo fluorescence diffuse optical tomography of breast cancer in humans. Opt Express 2007, 15:6696-6716

33. Ntziachristos V, Yodh AG, Schnall M, Chance B: Concurrent MRI and diffuse optical tomography of breast after indocyanine green enhancement. Proc Natl Acad Sci U S A 2000, 97:2767-2772

34. Mathur S, Vohra RS, Germain SA, Forbes S, Bryant ND, Vandenborne K, Walter GA: Changes in muscle T2 and tissue damage after downhill running in mdx Mice. Muscle Nerve 2011, 43:878-886

35. Yaseen MA, Yu J, Jung B, Wong MS, Anvari B: Biodistribution of Encapsulated Indocyanine Green in Healthy Mice. Mol Pharm 2009, 6: $1321-1332$

36. Warren GL, Hayes DA, Lowe DA, Williams JH, Armstrong RB: Eccentric contraction-induced injury in normal and hindlimbsuspended mouse soleus and EDL muscles. J Appl Physiol (1985) 1994, 77:1421-1430

37. Psatha M, Wu Z, Gammie FM, Ratkevicius A, Wackerhage H, Lee JH, Redpath TW, Gilbert FJ, Ashcroft GP, Meakin JR, Aspden RM: A longitudinal MRI study of muscle atrophy during lower leg immobilization following ankle fracture. J Magn Reson Imaging 2012, 35:686-695

38. Caron AZ, Drouin G, Desrosiers J, Trensz F, Grenier G: A novel hindlimb immobilization procedure for studying skeletal muscle atrophy and recovery in mouse. J Appl Physiol 2009, 106:2049-2059

39. Kraft JC, Ho RJ: Interactions of indocyanine green and lipid in enhancing near-infrared fluorescence properties: the basis for nearinfrared imaging in vivo. Biochemistry 2014, 53:1275-1283

40. Sheng Z, Hu D, Zheng M, Zhao P, Liu H, Gao D, Gong P, Gao G, Zhang P, Ma Y, Cai L: Smart human serum albumin-indocyanine green nanoparticles generated by programmed assembly for dualmodal imaging-guided cancer synergistic phototherapy. ACS Nano 2014, 8:12310-12322

41. Možina H: Near-infrared spectroscopy for evaluation of global and skeletal muscle tissue oxygenation. World J Cardiol 2011, 3:377 